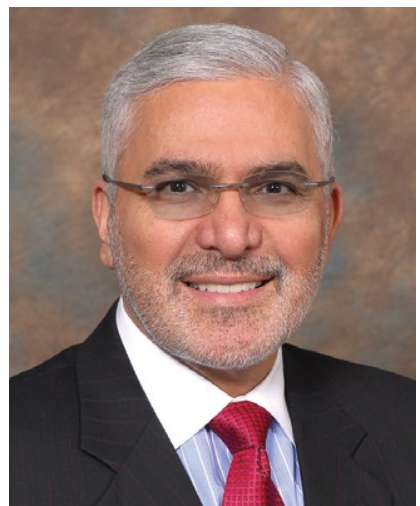

Henry A. Nasrallah, MD Editor-in-Chief

doi: $10.12788 /$ cp. 0105

\section{Rapid advances in understanding the many etiologies and subtypes of the heterogenous mood and psychotic disorders are invalidating the notion of treatment resistance}

\title{
Treatment resistance is a myth!
}

For millennia, serious psychiatric brain disorders (aka mental illnesses, melancholia, madness, insanity) were written off as incurable, permanent afflictions. It's no wonder that they were engulfed with the stigma of hopelessness.

But then came the era of serendipitous discoveries in the mid-20th century, with the felicitous arrival of antipsychotics, antidepressants, and lithium. The dogma of untreatability was shattered, but in its wake, the notion of treatment resistance emerged, and promptly became the bane of psychiatric clinicians and the practice of psychopharmacology.

Many patients with mood and psychotic disorders responded to the medications that were introduced in the 1950s and 1960s, but some either derived partial benefit or did not improve at all. These partial or poor responders were labeled "treatment-resistant," and caring for them became a major challenge for psychiatric physicians that continues to this day. However, rapid advances in understanding the many etiologies and subtypes of the heterogeneous mood and psychotic disorders are invalidating the notion of treatment resistance, showing it is a fallacy and a misnomer. Let's examine why.

\section{Treatment-resistant depression (TRD)}

Psychiatric clinics and hospitals are clogged with patients who do not respond to $\geq 2$ evidence-based antidepressants and carry the disparaging label of "TRD." But a patient manifesting what appears to be major depressive disorder (MDD) may actually have one of several types of depression that are unlikely to respond to an antidepressant, including:

- iatrogenic depression due to a prescription medication

- depression secondary to recreational drug use

- depressive symptoms secondary to a general medical condition

- bipolar depression.

Thus, a significant proportion of patients diagnosed with MDD are labeled TRD because they do not respond to standard antidepressants, when in fact they have been misdiagnosed and need a different treatment.

Even when the diagnosis of MDD is accurate, psychiatric neuroscience advances have informed us that MDD is a heterogeneous syndrome with multiple "biotypes" that share a similar phenotype. ${ }^{1,2}$ In the past, TRD has been defined as a failure to respond to $\geq 2$ adequate trials (8 to 12 weeks at a maximum tolerated dose) of antidepressants from different classes (such as tricyclic or heterocyclic antidepressants, selective serotonin reuptake inhibitors, or serotonin-norepinephrine reuptake inhibitors). For decades, patients with TRD have been referred to electroconvulsive therapy (ECT), and have experienced an excellent response rate. So TRD is in fact an artificial concept and 
term, applied to a subtype of MDD that does not respond to standard antidepressants, but often responds very well to neurostimulation (ECT and transcranial magnetic stimulation [TMS]).

When an antidepressant is approved by the FDA based on "successful" placebo-controlled double-blind trials, there is always a subset of patients who do not respond. However, the success of a controlled clinical trial is based on a decline in overall mean depression rating scale score in the antidepressant group compared with the placebo group. Not a single antidepressant has ever exerted full efficacy in $100 \%$ of patients who received it in an FDA trial because the sample is always a heterogeneous mix of patients with various depression biotypes who meet the DSM clinical diagnosis of MDD. Most often, only approximately $50 \%$ do, which is enough to be statistically significantly better than the roughly 30\% response rate in the placebo group. It is impossible for a heterogeneous syndrome comprised of biologically different "diseases" to respond to any single medication! Patients who do not respond to an antidepressant medication that works in other patients represent a different subtype of depression that is not TRD. Biotypes of the depression syndrome have different neurochemical underpinnings and may respond to different mechanisms of therapeutic action, yet to be discovered.

A very common clinical mistake occurs when patients with bipolar depression are misdiagnosed as having MDD because most of them experience depression as their initial mood episode. These patients often end up being classified as having TRD because bipolar depression very frequently fails to respond to several of the antidepressants that are FDA-approved for MDD. When these patients are correctly diagnosed, many will respond to one of the medications specifically approved for bipolar depression that were launched over the past 15 years (quetiapine, lurasidone, and cariprazine). However, bipolar disorder is also a heterogeneous spectrum, and some patients with bipolar depression may fail to respond to any of these 3 medications and are promptly regarded as TRD. Such patients often respond to neuromodulation (TMS, ECT, or vagus nerve stimulation [VNS]), indicating that they may have a different type of bipolar depression, such as bipolar type II.

A more recent example of the falsehood of TRD as a spurious diagnosis is the dramatic and rapid response of patients who are chronically depressed (both those with MDD and those with bipolar depression) to ketamine infusions. ${ }^{3,4}$ Responders to ketamine, a glutamate $\mathrm{N}$-methyl-D-aspartate (NMDA) receptor antagonist, prove that nonresponders to monoamine reuptake inhibitors must not be falsely labeled as having TRD. They have a different subtype within the depression syndrome that is mediated by glutamatergic pathways, instead of monoamines such as serotonin, norepinephrine, or dopamine. In addition, unlike monoaminergic antidepressants, NMDA antagonists rapidly reverse suicidal urges, above and beyond rapidly reversing chronic, so-called TRD.

In the same vein, numerous reports have shown that buprenorphine has significant efficacy in TRD (and suicide urges, as does ketamine), which implicates opioid pathways as mediating some subtypes of TRD. ${ }^{5}$ The monoamine model of depression, which dominated the field and dragged on for half a century, has distracted psychiatric researchers from exploring and recognizing the multiple neurochemical and neuroplastic pathways of the depression syndrome, thus falsely assuming that depression is a
Editorial Staff

EDITOR Jeff Bauer

SENIOR EDITOR Sathya Achia Abraham ASSISTANT EDITOR Jason Orszt WEB EDITOR Kathryn Wighton

Art \& Production Staff

CREATIVE DIRECTOR Louise Koenig

ART DIRECTOR Pat Fopma

DIRECTOR, JOURNAL MANUFACTURING Michael Wendt

PRODUCTION MANAGER Donna Pituras

Publishing Staff

PUBLISHER Sharon Finch

DIRECTOR EBUSINESS DEVELOPMENT Alison Paton

SENIOR DIRECTOR OF SALES

Tim LaPella

Editor-in-Chief Emeritus

James Randolph Hillard, MD

Frontline Medical Communications VP, SALES Mike Guire

VP, DIGITAL CONTENT \& STRATEGY Amy Pfeiffer

PRESIDENT, CUSTOM SOLUTIONS JoAnn Wahl

CIRCULATION DIRECTOR Jared Sonners DIRECTOR, CUSTOM PROGRAMS Patrick Finnegan

In affiliation with Global Academy for Medical Education, LLC

PRESIDENT David J. Small, MBA

\section{FRONTLNE MLedge}

7 Century Drive, Suite 302

Parsippany, NJ 07054

Tel: (973) 206-3434

Fax: (973) 206-9378

www.frontlinemedcom.com

Subscription Inquiries: subscriptions@mdedge.com

Published through an educational partnership with 
The rapid growth of biomarkers will usher in an era of precision psychiatry that will eliminate the term 'treatment resistance' monolithic disorder that responds to elevating the activity of brain monoamines. This major blind spot led to the ersatz concept of TRD.

\section{Treatment-resistant schizophrenia (TRS)}

Since the discovery of chlorpromazine and other antipsychotics in the 1950s, it became apparent that a subset of patients with schizophrenia do not respond to medications that block dopamine D2 receptors. Partial responders were labeled as having TRS, and complete nonresponse was called refractory schizophrenia. Many patients with severe and persistent delusions and hallucinations were permanently hospitalized, and unable to live in the community like those who responded to dopamine antagonism.

In the late 1980s, the discovery that clozapine has significant efficacy in TRS and refractory schizophrenia provided the first insight that TRS and refractory schizophrenia represent different neurobiologic subtypes of schizophrenia. ${ }^{6,7}$ The extensive heterogeneity of schizophrenia (with hundreds of genetic and nongenetic etiologies) is now widely accepted. ${ }^{8}$ Patients with schizophrenia who do not respond to dopamine receptor antagonism should not be labeled TRS, because they can respond to a different antipsychotic agent, such as clozapine, which is believed to exert its efficacy via glutamate pathways.

But what about the $50 \%$ of patients with TRS or refractory schizophrenia who do not respond to clozapine? ${ }^{9}$ They do not have TRS, either, but represent different schizophrenia biotypes that may respond to other medications with different mechanisms of action, such as lamotrigine, ${ }^{10}$ which is a glutamate modulator; pimavanserin, ${ }^{11}$ which is an inverse agonist of the serotonin 5HT-2A receptor; allopurinol, ${ }^{12,13}$ an adenosine modulator; or estrogen, ${ }^{14}$ a neurosteroid. Future research will continue to unravel the many biotypes of the highly heterogeneous schizophrenia syndrome that are "nondopaminergic" and do not respond to the standard class of dopamine antagonists (previously called neuroleptics and now known as antipsychotics). ${ }^{15}$ Future treatments for schizophrenia may depart from modulating various neurotransmitter receptors to targeting entirely different neurobiologic processes, such as correcting mitochondria pathology, inhibiting microglia activation, repairing white matter, reversing apoptosis pathways, inducing neuroplasticity, arresting oxidative stress and inflammation, and other neuroprotective mechanisms.

The rapid growth of biomarkers in psychiatry ${ }^{16}$ will usher in an era of precision psychiatry ${ }^{17}$ that will eliminate the term "treatment resistance." Our psychiatric practice will then benefit from "canceling" this demoralizing and clinically unjustified term that has needlessly fostered therapeutic nihilism among psychiatric physicians.

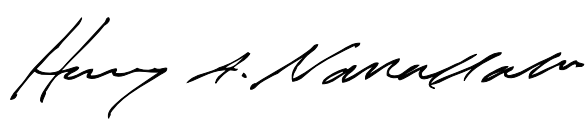

\section{Henry A. Nasrallah, MD}

Editor-in-Chief

References

1. Milaneschi Y, Lamers F, Berk M, et al. Depression heterogeneity and its biological underpinnings: toward immunometabolism depression. Biol Psychiatry. 2020;88(5):369-380.

2. Akiskal HS, McKinney WT Jr. Overview of recent research in depression. Integration of ten conceptual models into a comprehensive clinical frame. Arch Gen Psychiatry. 1975;32(3):285-305

3. Zarate CA Jr. Ketamine: a new chapter in antidepressant development. Brazilian J Psychiatry. 2020;42(6):581-582.

4. Diazgranados N, Ibrahim L, Brutsche NE, et al. A randomized add-on trial of $\mathrm{N}$-methyl-D-aspartate antagonist in treatment-resistant bipolar depression. Arch Gen Psychiatry. 2010;67(8):793-802

5. Serafini $G$, Adavastro G, Canepa G, et al. The efficacy of buprenorphine in major depression, treatmentresistant depression and suicidal behavior: a systematic review. Int J Mol Sci. 2018;19(8):2410. 


\section{From the Editor}

continued from page 16

6. Potkin SG, Kane JM, Correll CU, et al. The neurobiology of treatment-resistant schizophrenia: paths to antipsychotic resistance and a roadmap for future research. NPJ Schizophr. 2020;6(1):1.

7. Campana M, Falkai P, Siskind D, et al. Characteristics and definitions of ultra-treatment-resistant schizophrenia - a systematic review and meta-analysis. Schizophr Res. 2021;228:218-226.

8. Kinon BJ. The group of treatment resistant schizophrenias. Heterogeneity in treatment-resistant schizophrenia (TRS). Front Psychiatry. 2019; 9:757.

9. Siskind D, Siskind V, Kisely S. Clozapine response rates among people with treatment-resistan schizophrenia: data from a systematic review and meta-analysis. Can J Psychiatry. 2017;62(11): 772-777.

10. Tiihonen J, Wahlbeck K, Kiviniemi V. The efficacy of lamotrigine in clozapine-resistant schizophrenia: a systematic review and meta-analysis. Schizophr Res. 2009;109(1-3):10-14

11. Nasrallah HA, Fedora R, Morton R. Successfu treatment of clozapine-nonresponsive refractory hallucinations and delusions with pimavanserin a serotonin 5HT-2A receptor inverse agonist. Schizophr Res. 2019;208:217-220.

12. Linden N, Onwuanibe A, Sandson N. Rapid resolution of psychotic symptoms in a patient with schizophrenia using allopurinol as an adjuvant: a case report. Clin Schizophr Relat Psychoses. 2014;7(4):231-234.

13 Lintunen $\mathrm{J}$, Lähteenvuo $\mathrm{M}$, Tiihonen $\mathrm{J}$, et al Adenosine modulators and calcium channe blockers as add-on treatment for schizophrenia. NPJ Schizophr. 2021;7(1):1.

14. Kulkarni J, Butler S, Riecher-Rössler A. Estrogens and SERMS as adjunctive treatments for schizophrenia. Front Neuroendocrinol. 2019;53:100743. doi 10.1016/j.yfrne.2019.03.002

15. Tandon R, Nasrallah HA, Keshavan MS Schizophrenia, "just the facts" 5. Treatment and prevention. Past, present and future. Schizophr Res. 2010;122(1-3):1-23.

16. Nasrallah HA. Biomarkers in neuropsychiatric disorders: translating research to clinical applications. Biomarkers in Neuropsychiatry. 2019;1:100001. doi: 10.1016/j.bionps.2019.100001

17. Nasrallah HA. The dawn of precision psychiatry Current Psychiatry. 2017;16(12):7-8,11 